\title{
Untersuchungen über badischen Schaumkalk
}

\author{
Stratigraphischer Teil.
}

\author{
Inaugural-Dissertation \\ zur Erlangung der Doktorwürde \\ der Hohen \\ Naturwissenschaftlich-Mathem. Fakultät \\ der Ruprecht-Karls-Universität \\ $2 \mathrm{uHeidelberg}$
}

vorgelegt von

Hildegard Barg

aus Berlin.

193.3. 


\title{
Untersuchungen über Badischen Schaumkalk.
}

\author{
Stratigraphischer Teil.
}

Die Arbeit wurde ausgeführt auf Grund der Anregungen der Herren Geh. Hofrat Prof. Dr. Salomon-Calvi und Prof. Dr. Rüger in Geologischen Institut der Universität Heidelberg. Meinen Lehrern bin ich für die mir zuteil gewordene Förderung und jederzeit bereitwillig erteilten Rat zu großem Dank verpflichtet.

Die Untersuchungen wurden abgedruckt im Jahresbericht des Obherrhein. Geolog. Vereins für das Geschäftsjahr 1932/33, Jahrgang: I933, Bd, XXII (NF).

Es wurde festgestellt, daß die beiden unteren Schaumkalkbänke des Baulandes sich in den beiden im Kraichgau bekannten Schaumkalkbänken fortsetzen, und dalß sie den beiden unteren Schatumkalkbänken Mitteldeutschlands, bezw. Unterfrankens, entsprechen. Die dritte, oberste Schaumkalkbank ist in Baden lediglich an der Tauber und im nördlichen Bauland ausgebildet. Sie stellt eine lokale Erscheinung clar, die ihre Entstehung einer in flachem Wasser befindlichen Barre (Vollratu's Tauberbarre) verdankt.

Eine ausführliche Darstellung des mikroskopischen Befundes, sowie einige theoretische Erörterungen zur Paläogeographie werden im nächsten Jahresbericht des Oberrh. G. V. abgedruckt. Es sei jedoch hier schon daratuf hingewiesen, daß sich zwischen der petrographischen Ausbildung der Bänke und der Art ihres Ablagerungsraumes innerhalb des Wellenkalkbeckens eine klare Abhängigkeit aufweisen ließ.

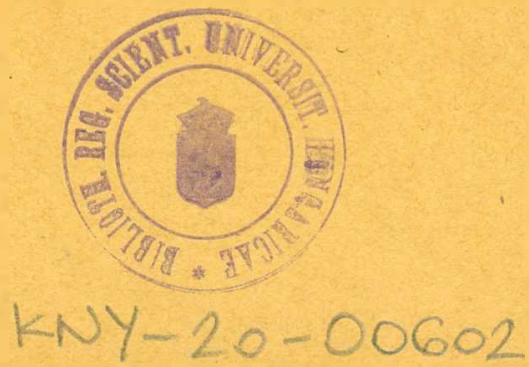




\section{Lebenslauf.}

Ich, $\mathrm{Hildegard} \mathrm{Barg}$, wurde am 8. November rgo4 zu Berlin als Tochter des Gastwirtes Reinhold Barg geboren und besitze preußische Staatsangehörigkeit. Ich besuchte die Klinkhardtsche Privatschule in Berlin, später das städtische Lyzeum zu Görlitz, das ich I92I mit dem Reifezeugnis cler I. Klasse verließ. Nach mehreren Jahren kaufmännischer Tätigkeit in Köln a. Rh. legte ich im September 1926 das Abiturientenexamen an der Gerhart-Hauptmann-Oberrealschule z11 Breslau als Schulfremde ab. Ich studierte darauf 5 Semester in Heidelberg, 2 Semester in Berlin, und abermals 3 Semester in Heidelberg. Als Studienfächer wählte ich Geologie, Biologie und Chemie, und nahm dazu später Geographie und Philosophie auf. Im Dezember I93I bestand ich die Prüfung für das wissenschaftliche Lehramt an höheren Schulen in Baden. (Hauptfücher Geologie mit Mineralogie, Geographie. Nebenfach- Biologie. Zusatzfach: Philosophie.)

Meine akademischen Lehrer waren

in Heidelberg: Erdmannsdörffer, Faust, Freudenberg, Glück, Herlust, Hoffmann, Jaspers, Jost, Kinzl, Lenard, Merton, Röhrer, Rüger, Salomon-Calvi, Sölch, Spek.

in Berlin: Belowsky, v. Haller, Hesse, Johnsen, Kniep, Markgraf, Markus, P'ompeckj, Schwemmle. 
